\title{
Challenges of implementing an Integrated Disease Surveillance and Response strategy using the current Health Management Information System in Tanzania
}

\author{
J. M. MGHAMBA, L.E.G. MBOERA, W. KREKAMOO, K.P. SENKORO, \\ S.F. RUMISHA, E. SHAYO \& P. MMBUJI \\ National Institute for Medical Research \\ Integrated Disease Surveillance and Response Project \\ P.O. Box 9653, Dar es Salaam, Tanzania
}

\begin{abstract}
Tanzania adopted an Integrated Disease Surveillance and Response (IDSR) strategy in 1998 in order to strengthen its infectious disease surveillance system. During that time, the country had 5 separate surveillance systems to monitor infectious disease trends and disease control programmes. The systems included the Health Management Information System (HMIS); Infectious Disease Week Ending; Tuberculosis/Leprosy; Human Immunodeficiency Virus/Acquired Immunodeficiency Syndrome; and Acute Flaccid Paralysis/Poliomyelitis). An assessment of the surveillance systems in the country has shown inadequacy in the use of standard case definitions and laboratory confirmation of cases, supervision and feedback as well as data collection tools. Moreover, in some of the existing vertical disease surveillance programmes, the epidemiological data generated were neither fully analyzed nor utilized at district level, but rather were forwarded to higher levels, which hardly provided any feedback to the lower levels. For these and other reasons, the Ministry of Health adopted the IDSR strategy that will focus on selected priority diseases. Although a significant progress has been made as far as the IDSR strategy is concerned, its implementation is facing several challenges. This is due to the fact that the current surveillance system has to utilize the existing HMIS system in collecting and compiling epidemiological data from health facility and district levels. This paper intends to discuss in detail challenges, which the Ministry of Health and district councils have to take into consideration during the implementation of IDSR strategy. Recommendations are also made for possible adoption to enable smooth running of the IDSR strategy at the national, district and facility levels.
\end{abstract}

\section{Introduction}

In simple terms, public health surveillance can be defined quite succinctly as generation of information for action. This includes an on-going systematic collection, analysis, interpretation, and dissemination of health data. Data collected through this process are used to assist in detection and tracking of diseases and hence help health teams to promptly respond to outbreaks, set priorities, plan interventions and, mobilize and allocate resources.

The surveillance of infectious diseases has recently assumed greater importance in most countries because of emerging and re-emerging of infectious diseases and also because strains of pathogens causing tuberculosis (TB), malaria, cholera, dysentery, and pneumonia have developed resistance to common and cheap antimicrobial drugs.

Since 1998, surveillance of the infectious diseases in Tanzania has been under five separate systems namely Health Management Information System (HMIS); Infectious Disease Week Ending (IDWE); TB/Leprosy; Human Immunodeficiency Virus/ Acquired Immunodeficiency Syndrome (HIV/AIDS) and Acute Flaccid Paralysis (AFP)/Poliomyelitis. While HMIS and IDWE cover a wide range of diseases (Mboera et al., 2001a), the other three surveillance systems are disease specific, vertically and donor oriented. The implementation of the systems starts from health facility level upwards.
The HMIS was introduced in Tanzania between 1993 and 1997 to replace the old system known as the Health Information System (HIS). The introduction of HMIS followed the realisation that the former system was inadequate and inappropriate to the growing information needs of the health sector. The former system was also fragmented in nature with bottom-up flow of information and with little or no feedback to the lower levels. There was also little capacity for analysis, interpretation and use of the data at various levels of the health care delivery system (Mwangu and Otito, 2000). However, the HMIS covers the entire spectrum of the health system from the health facility, district, regional, to the national levels. At the health facility level (dispensary, health centre and hospital), HMIS is made up of various registers, tally sheets and a data entry book, which is used to consolidate all data. Through simple calculations, basic performance indicators are derived and the health facility management is required to take action using these indicators. The HMIS system includes 12 "books" of forms, registers, and reports that health workers use to report all types of diseases and health services. In addition, the books require health workers to compile data in some basic ways for analysis: by age, gender, top 10 diseases, etc.

With the use of HMIS, data from health facilities are reported quarterly to the District Medical Office (DMO) through forms contained in the Report Book. At the district level, the main HMIS tool is the District 
Data Processing File (DPF). The DPF is used to process data from all health facilities within the district. However, emergency reports including, disease outbreaks are sent directly to the DMO for immediate action using separate specific forms. The DMO then reports to the Regional Medical Office (RMO) by filling out special forms contained in the DPF. At the regional level, HMIS is computerized and data from the districts are fed into a computer. The RMO reports to the Ministry of Health where HMIS is also fully computerized and data received are fed into the national HMIS database.

There are only a few studies that have evaluated the HMIS since its inception in 1993. When assessing the structure and performance of the different surveillance systems, Nsubuga et al. (2002) found out that HMIS was well covered in health facilities, district and region levels. The other four surveillance systems were found at $<20 \%$ of health facilities and $<75 \%$ of district medical offices. In another field study, it has also been indicated that HMIS implementation has been successful in the sense that all regions in Tanzania Mainland were using it (Mwangu and Otito, 2000), although at varying extents.

Despite some successes in the vertical surveillance systems, several challenges exist. It has been observed that none of the five surveillance systems is adequately being implemented and none of the core or support activities are adequately performed at any level of the health care system (Nsubuga et al., 2002). In addition, HIMS has been hampered by problems of inadequate staffing and inadequate provision of medical supplies (Mwangu and Otito, 2001) as well as lack of sustainability in training of health workers in its use. In some health facilities, data collection and analysis tools are not regularly used as required and as a result these factors affect the quality and quantity of data collected, analyzed and reported to the appropriate levels. Other challenges facing these disease surveillance systems have been cited as failure to report diseases of epidemic potential in time, incomplete reporting of notifiable diseases, failure to use available information to follow disease trends, inadequate laboratory involvement in case detection and poor feedback to health workers and communities. In some cases, the presence of vertical programmes resulted into duplication of resources meant for surveillance and response. It has been reported recently that there is underutilization of surveillance information in decision-making at the district level such that the data generated are neither analysed nor utilized but rather forwarded to higher levels with hardly any feedback (Mboera et al., 2001a).

Recognizing and addressing these problems, Tanzania adopted a proposal by WHO/AFRO at its $48^{\text {th }}$ meeting in August 1998, of establishing an Integrated Disease Surveillance and Response (IDSR) strategy. In this strategy, several activities from the different vertical programmes are coordinated and streamlined in order to make best use of scarce resources. It is envisaged that embracing the integrated disease surveillance strategy could surmount some of the challenges described above (Mboera et al., 2001b).

Since its inception, the implementation of IDSR strategy, specifically, data collection and management has been relying on the existing HMIS tools. Patient registers have remained the same. It was the objective of this study to conduct an assessment of the infectious disease surveillance system in the country in relation to data management tools and identify enabling and constraining factors in its implementation. This paper intends to discuss in detail the challenges within the surveillance functions, which the Ministry of Health and district councils have to take into consideration during the implementation of IDSR strategy when using the current surveillance system. Recommendations are also made for possible adoption to enable smooth running of the IDSR strategy.

\section{Materials and methods}

A situation analysis was conducted in Babati, Mbulu, Dodoma Rural and Mpwapwa Districts. The overall purpose of the analysis was to better understand how the implementation of IDSR using the existing surveillance systems function and identify the most important actions needed to improve disease surveillance and response strategy. The situation analyses were carried out in Babati and Dodoma Rural Districts in April and August 2002, respectively and in Mpwapwa and Mbulu in April 2003. Data were collected through two main mechanisms:

i.) Quantitative data through documentary review;

ii.) Qualitative data through individual

interviews with health staff.

Qualitative survey was done to gain a strong understanding of exactly how and why disease surveillance-related activities occur and about perceptions of surveillance from the perspective of those who are directly responsible for implementation. This type of information is important for deciding on priorities and making design decisions about strategies and interventions.

Quantitative data were collected from the Office of the District Medical Officer on such topics as surveillance (diseases reported, completeness and timeliness of reporting to district and regional levels) and management information (e.g., staffing, data management and utilization, feedback and supervisions). The quantitative data obtained provided additional descriptive information important for the design of activities.

Eight of the 36 health facilities in Babati and 13 of the 72 health facilities in Dodoma Rural were 
visited. They were selected to represent different types of health facilities (hospital, health centre, and dispensary); urban and rural context; geographic variation throughout each district; and at least one nongovernmental facility per district. In both Babati and Dodoma Rural, at least one facility was selected from each division. Five health facilities were assessed in Mbulu and Mpwapwa districts.

\section{Results}

The identified key findings for the IDSR strategies are summarised in Table 1 and 2.

Table 1: Constraints in disease surveillance in Dodoma Rural, Babati, Mbulu and Mpwapwa districts

\section{SURVEILLANCE FUNCTION CURRENT SITUATION}

Detection using standard case definition

Recording

Reporting

Data analysis
-Some Health Workers use knowledge from training, skills, experience, but level varies

-SCD of 13 IDSR priority diseases and other guidance materials insufficiency at facility

-Inadequate tools for diagnosis

-Many cases of non-epidemic prone diseases treated elsewhere than at Health Facility

-Some diagnoses and related data were not recorded by clinicians and not all health staff record all cases into HMIS or Case Investigation forms

-Inconsistent way of recording diagnoses (some use etiologic agents instead of name of disease)

-Tally sheets not always available or used (more work, no benefit to person doing tally)

-Registers lack room for recording other important information (Register Book 5 for out-patients lacks an update on confirmed diagnosis after results from laboratory are given)

-No recording of new dates at start of each day

-Lack of Inpatient Registers (Guidelines exist as to what to include in the registers as such the health facilities do improvise themselves registers according to their needs leading to unstandardized inpatient registers)

-Information on referral patients is limited to tallying

-No system for recording information on most of diseases as pertaining to deaths and other outcomes in the communities

-No supervisory support for ensuring accurate recording

-From outbreak treatment camps data are sent directly to district, bypassing the respective facility

-Lack of motivation to complete and report for HMIS

-Limited means for verbal communications for reporting

- Incomplete and late reporting

-Limited collaboration between sectors and vertical programmes for reporting

-Some workers do not understand the value of zero reporting

-Lack of reporting forms (weekly and monthly) in some health facilities

-Staff perception of inadequate time for compiling and reporting

-Little or no evidence was found in analysis of IDSR data, at both district and facility level.

-Some health staff have never received any training on HMIS

-Perceived value of analysis for making good decisions inadequate

-Lack of monitoring of epidemiological parameters with the existing HMIS

system

- No clear or defined mechanism for feedback 


\section{Identification and recording of cases}

There was adequate provision of HMIS tools for data collection and reporting in terms of registers and tally sheets.

Reporting of monthly IDSR requires separation of severe and mild pneumonia and diarrhoea in terms of degree of dehydration (some and severe dehydration). These separations are not accommodated in the current HMIS health facility register. The HMIS Book 5 (used for outpatient registration) had no space for recording of or modifications in diagnosis (that might result from further laboratory testing). It was observed that some facilities do not record the outcome of an admitted patient (whether discharged, absconded or died) (Table $1,2)$ as this space is not accommodated in the register. Since there were no Standard Case Definitions for most diseases, clinicians relied on their clinical training leading to inconsistency in recording of diagnosis. In both districts, most providers interviewed had a fair clinical understanding of the 13 IDSR diseases. However, there was no consistency in the level of understanding across health workers. In addition, although some providers record the information at the time of the consultation, others do not. Many providers complained about the time required to record all the HMIS information properly. Some providers have not received any training on their HMIS responsibilities.

\section{Compiling and reporting of facility level information}

The reporting system in HMIS is done quarterly, while IDSR requires weekly and monthly reporting. Health facilities are required to immediately notify the District Medical Officer when they suspect an outbreak, and to provide week-ending reports for 7 diseases. Many facility staff in both districts complained of the burden of compiling and submitting weekly, monthly and quarterly reports. In some facilities, however, members of staff worked together as a team to complete their reports.

\section{Data management and utilization for decision making}

Little or no evidence was found in either district regarding analysis of surveillance data, at both district and facility level. Some routine analysis of HMIS data was observed, such as identification of top 10 diseases (as ranked by the number of consultations). Such analysis was embedded in the HMIS annual reporting forms. IDRS requires epidemiological data analysis by place, time and person. Few facilities in the districts had carried out simple analyses but skills and appreciation of purpose of analysis varied. In Babati, several facility level staff noted that analysis is for those with more training, and that the task is too sophisticated for them. At district level in Dodoma and Babati, some limited place and person analysis was done during outbreaks, but lack of clear formats and expectations have led to analysis being given a low priority. Both districts had computers at the district office, but they were not used for routine data analysis. The majority of health personnel are lacking computer knowledge and skills especially in relation to data processing and analysis.

\section{Providing feedback and supervision}

Although some kind of data analysis was performed at district level as required by HMIS, no feedback was provided to the facility or community level as required by IDSR. Even during supervisory visits facilities were not given feedback on the analyzed data that showed trends and patterns for different diseases in their districts.

\section{Monitoring and Evaluation}

There was lack of monitoring of epidemiological parameters with the existing HMIS system. Although data was reported weekly, monthly and quarterly, monitoring of data collection and reporting was not done in all districts for either of the two surveillance systems despite the fact that each district had HMIS focal person. The HMIS appeared to be well-followed by health workers in the four districts. 
Table 2: The inconsistence between HMIS and IDSR system

\begin{tabular}{|c|c|c|}
\hline Item & HMIS & IDSR \\
\hline Weekly tally & Include Relapsing fever & $\begin{array}{l}7 \text { diseases excluding relapsing fever } \\
\text { (cholera, acute flaccid paralysis, } \\
\text { measles, cerebro-spinal meningitis, } \\
\text { plague, yellow fever, rabies and animal } \\
\text { bites) }\end{array}$ \\
\hline Monthly tally & 11 reportable diseases & 13 reportable disease \\
\hline Registers & $\begin{array}{l}\text { Contains several registers: one for inpatient, } \\
\text { and one for outpatient, and separate ones } \\
\text { for other services e.g. Immunisation, } \\
\text { Reproductive and Child Health }\end{array}$ & $\begin{array}{l}\text { Report for IDSR diseases mainly } \\
\text { utilized the inpatient and outpatient } \\
\text { registers }\end{array}$ \\
\hline OPD register & $\begin{array}{l}\text { The outpatient register includes separate } \\
\text { pages for the } 11 \mathrm{HMIS} \text { reportable diseases. }\end{array}$ & $\begin{array}{l}\text { IDSR requires reporting of } 13 \text { priority } \\
\text { disease monthly }\end{array}$ \\
\hline Attendance dates & $\begin{array}{l}\text { There is no space for attendance dates in } \\
\text { the OPD register. Instead, each page has a } \\
\text { "beginning" date. }\end{array}$ & $\begin{array}{l}\text { IDSR forms require weekly reporting } \\
\text { from one date to another) }\end{array}$ \\
\hline Recording of death & $\begin{array}{l}\text { There is no space to indicate deaths or other } \\
\text { outcomes in the OPD register. }\end{array}$ & $\begin{array}{l}\text { IDSR forms require reporting of } \\
\text { deaths }\end{array}$ \\
\hline Laboratory results & $\begin{array}{l}\text { There is no space for laboratory results or } \\
\text { change in diagnosis in the OPD register. }\end{array}$ & $\begin{array}{l}\text { IDSR requires that health } \\
\text { workers seek laboratory confirmation. }\end{array}$ \\
\hline Monthly reports & $\begin{array}{l}\text { The HMIS monthly reports "bacterial" } \\
\text { diarrhoeal diseases } \\
\text { The HMIS monthly reports pneumonia for } \\
\text { inpatients }\end{array}$ & $\begin{array}{l}\text { IDSR forms report "diarrhea with } \\
\text { some/severe dehydration } \\
\text { IDSR forms report "pneumonia" and } \\
\text { "severe pneumonia" separately for } \\
\text { inpatients }\end{array}$ \\
\hline
\end{tabular}

\section{Discussion}

Adopting and implementing IDSR using the existing HMIS involves major improvement of the core or supporting functions of the current system. In this situation analysis, in all the four districts there was inadequate use of standard case definitions, data collection tools, laboratory confirmation of cases, poor quality of reporting, inadequate data analysis, poor supervision and feedback. Previously, in assessing the structure and performance for five infectious disease surveillance systems in Tanzania Nsubuga et al., (2002) found that standardized case definitions were used for only $14.3 \%$ (3/21) of the infectious diseases. In our findings, health workers relied mostly on their clinical skills rather than applying standard case definition leading to inconsistent recording of diagnoses. This is an important finding with regard to proper detection of cases and hence inconsistence in recording in the HMIS registers.
In another study done on the challenges and future prospects of HMIS implementation at the district level in the context of health reforms, it was found out that despite its success, HIMS has been hampered by several challenges including data collection and analysis tools, which although present, were irregularly used. The understaffing, lack of skills by some health workers and motivation were found to explain the low utilization of data (Mwangu and Otito, 2000). Nsubuga et al. (2002), also found that although data analysis was carried out in all five surveillance systems, incidence and prevalence were rarely calculated and moreover trends in disease outbreaks were seldom tracked. Similar findings were observed in our study. This has an implication with regard to planning and taking action using evidence-based information and in this case the use of analysed data. Although this is currently emphasized under the IDSR strategy, analysis beyond ranking of top ten diseases is rarely done under the HMIS system at district level. 
Epidemiological data analysis and interpretation needs to be done at all levels of disease surveillance, right from community to national and international levels. The kind of analysis done at each level will vary according to requirements and what can be done at that particular level. At whatever level, data are normally analysed by time, place and person. Time analysis enables the comparison of cases reported for the current time (week/month/year) with the number received in the previous time (week/month/year). This enables the detection of any abrupt or long-term changes in disease occurrence. Whereas some functions of disease surveillance are only applicable to some levels, data analysis and interpretation is a requirement for all people involved in disease surveillance at all levels.

Although, to-date HMIS is available throughout the country, health data collection, analysis, reporting and utilization in disease surveillance is poor. The number of health facilities that utilize health information system is low. It has been realized that the major problem in our health system is lack of surveillance mechanism that should help districts to monitor epidemic prone diseases and other diseases of public health importance (Mboera et al., 2001a). Under the existing HMIS, weekly epidemiological reports are sent to the Epidemiology and Disease Control Section of the Ministry of Health, while the Quarterly epidemiological data are sent to the HMIS section of the Planning and Policy Department of the same Ministry. There is little collation of the same data resulting into variation in the reported disease data at the end of the year.

Feedback and supervision have also been found to be poor in this situation analysis. HMIS provide room for feedback, but was rarely done. Similar findings have been reported in a recent study by Nsubuga et al. (2002). One important tool for developing health workers and improving performance is the proper use of feedback and increase community compliance in outbreak control measures, the approach that is given much emphasis under the current IDSR strategy.

In conclusion, HMIS still serves as the main tool for IDSR data collection, and the tool has some areas that need to be modified or added to address IDSR data. Review of all data collection tool at both facility and district level should be done as well as coordination of data collection, processing and dissemination, training and supervision, monitoring and evaluation especially at district and facility levels. Establishment and support for common district data source should be done to ensure relevance of the data to the two levels. The recently introduced IDSR strategy by the Ministry of Health is away forward to the improvement of our routine health management information system (Mboera et al., 2001b, 2002). To address the identified problems, the current IDSR system, has developed additional data management tools, materials and resources to improve recording, reporting and data management at facility and district levels. The tools include the National IDSR Guidelines, reporting forms and District Analysis Book. However the tools are only been used in selected pilot districts.

The objective of IDSR strategy is to increase the availability of relevant, valued, and useful information by health providers and other identified information stakeholders at the district and facility levels. This will enable health providers to gain an understanding of IDSR and importance of surveillance-related data. It is important for each district and facility to realise that surveillance data are most useful for which actions for public health responses to base upon. It is expected that, with such provision of tools and capacity building that is been carried out by the National Institute for Medical Research (NIMR), the new IDSR strategy will be able to identify the most productive actions and approaches for information users to take for increasing the availability and use of quality information. This action is envisaged to improve district staff competency and knowledge of producing and demanding information related to disease surveillance, feedback, and operational actions.

Currently, NIMR in collaboration with the Ministry of Health is strengthening IDSR in 12 selected districts with the aim of addressing the issues of data management and information utilization. Information needs, indicators and their presentation format have been identified and developed to address IDSR actions, feedback, and management/operations 
needs, particularly at the district and facility levels. Other areas addressed in this programme include application of IDSR knowledge in the development of annual Comprehensive Council Health Plans. Development of appropriate procedures and information tools for facilitating collection of information for relevant application by health providers and other identified information stakeholders at the district and facility levels is emphasised under this programme. Monitoring and evaluation is part of the programme and is done quarterly. All these are done through revision of existing tools and the development of new tools and through capacity building among members of the CHMT and health facility staff.

It is the goal of the IDSR strategy to improve the timeliness of quality data for deploying a timely and appropriate response. The strategy aims at building a culture of information and improving health worker motivation for IDSR-related tasks by increasing the relevance, value, usefulness, and use of IDSR-related information at the facility and district levels and among other key local stakeholders. However, if this is to be achieved, the HMIS and IDSR systems need to be harmonised. This will ultimately provide the most effective design of data management and information flow and use for a sustainable and responsive surveillance system. It is time that the Ministry of Health should also clarify how these two systems can be integrated, to ease the burden of the health in both data management and reporting.

\section{References}

Mboera, L.E.G., Rumisha, S.F., Magesa, S.M. \& Kitua, A.Y. (2001a) Utilisation of Health Management Information System in disease surveillance in Tanzania. Tanzania Health Research Bulletin 3, (2), 15-18.

Mboera, L.E.G., Rumisha, S.F. \& Kitua, A.Y. (2001b) Strategic approach for strengthening national and regional disease surveillance system: The East African example. Tanzania Health Research Bulletin 3, (2), 6-9.

Mboera, L.E.G., Rumisha, S.F., Senkoro, K.P., Kamugisha, M.L. \& Kitua, A.Y. (2002) East African Integrated Disease Surveillance Network, Technical Report Submitted to Rockefeller Foundation, Dar es Salaam, Tanzania, July 2002.

Mwangu, M.A. \& Otito, C.K. (2000). HMIS implementation at the district level in the context of the Health Sector Reforms: Challenges and future prospects. Proceedings of the $18^{\text {th }}$ Annual Scientific Conference of the Tanzania Public Health Association, November 22-25, 1999, Dodoma, Tanzania. Pp 111-119.

Nsubuga, P., Eseko, N., Wuhib, T., Ndayimirije, N., Chungong, S. \& McNabb, S. (2002) Structure and performance of infectious disease surveillance and response, United Republic of Tanzania, 1998. Bulletin of the World Health Organization 80, 196-202. 